\title{
Seven Challenges for Communication in Modern Railway Systems
}

OPEN ACCESS

Edited by:

Wei Xu,

Southeast University, China

Reviewed by:

Nikolaos Nomikos,

University of the Aegean, Greece

Zhaohui Yang,

King's College London,

United Kingdom

${ }^{*}$ Correspondence:

Elias Yaacoub

elias.yaacoub@gmail.com

Specialty section:

This article was submitted to

Wireless Communications,

a section of the journal

Frontiers in Communications and

Networks

Received: 20 October 2020

Accepted: 08 December 2020

Published: 22 January 2021

Citation:

Yaacoub E, Alsharoa A, Ghazzai $H$ and Alouini M-S (2021) Seven Challenges

for Communication in Modern

Railway Systems.

Front. Comms. Net. 1:619527.

doi: 10.3389/frcmn.2020.619527

\author{
Elias Yaacoub ${ }^{1 *}$, Ahmad Alsharoa ${ }^{2}$, Hakim Ghazzai $^{3}$ and Mohamed-Slim Alouini ${ }^{4}$ \\ ${ }^{1}$ Computer Science and Engineering Department, Qatar University, Doha, Qatar, ${ }^{2}$ Electrical and Computer Engineering \\ Department, Missouri University of Science and Technology, Rolla, MO, United States, ${ }^{3}$ Stevens Institute of Technology, \\ Hoboken, NJ, United States, ${ }^{4}$ Computer, Electrical and Mathematical Sciences and Engineering (CEMSE) Division, King Abdullah \\ University of Science and Technology (KAUST), Thuwal, Saudi Arabia
}

To meet the increasing demands for passenger data rates, modern railway communication networks face significant challenges. The advent of $5 \mathrm{G}$ communications after the longterm evolution (LTE) and LTE-Advanced (LTE-A) systems provides several technological advances to address these challenges. In this paper, after reviewing the main 5G communication aspects for modern railways, we describe seven main challenges faced by train connectivity, and discuss appropriate solutions. Specifically, we elaborate on techniques for ensuring connectivity and energy efficiency for the passengers' user equipment (UE) through the use of mobile relays (MRs) on top of the train wagons in conjunction with intelligent resource allocation.

Keywords: railway communications, mobile relay, LTE, radio resource management (RRM), 5G

\section{INTRODUCTION}

With broadband wireless connectivity expected anytime anywhere, train passengers can no longer be served with the $3 \mathrm{G} / 4 \mathrm{G}$ base stations (BSs) of mobile operators deployed to meet the demand of the populated areas in the surroundings of the railroad track. Furthermore, with the increased requirements for efficient train control signaling, real-time transmission of mission-critical data, and accurate monitoring of the railroad status, GSM-R should evolve into the next-generation of wireless technologies (Friedner et al., 2018).

Moreover, railroad wireless communications coverage should include BSs dedicated for train passenger connectivity, in addition to, or co-located with, the BSs that are used for the train control network. The BSs can be connected to remote radio heads (RRHs) that are more densely deployed along the track, and communicating with a mobile relay (MR) located on top of the train (Yan et al., 2016). An enhanced performance is obtained when 2 MRs (front and rear) are located on top of the train, with several RRHs per BS deployed along the track (Kim et al., 2019). Another approach to increase coverage and capacity is the combination of macro BSs with small cells along the railroad track, with several MRs deployed on top of the train (Banerjee et al., 2018).

The role of MRs is essential in enhancing communications. They have outdoor antennas outside the train that are connected to the BS or RRH. With BSs/RRHs deployed along the railroad track, this outdoor connectivity is expected to be line of sight (LOS) most of the time. In addition, these MRs are usually connected to each other by fiber optic cables inside the train, and can thus distribute the received information to the user equipment (UEs) wirelessly using a dedicated network inside the train (e.g., WiFi).

In (Ghazzai et al., 2017), BSs were considered to be deployed along the track to ensure long range (LR) LOS connectivity to MRs, whereas 1 MR was deployed per wagon (i.e., several MRs per train), 


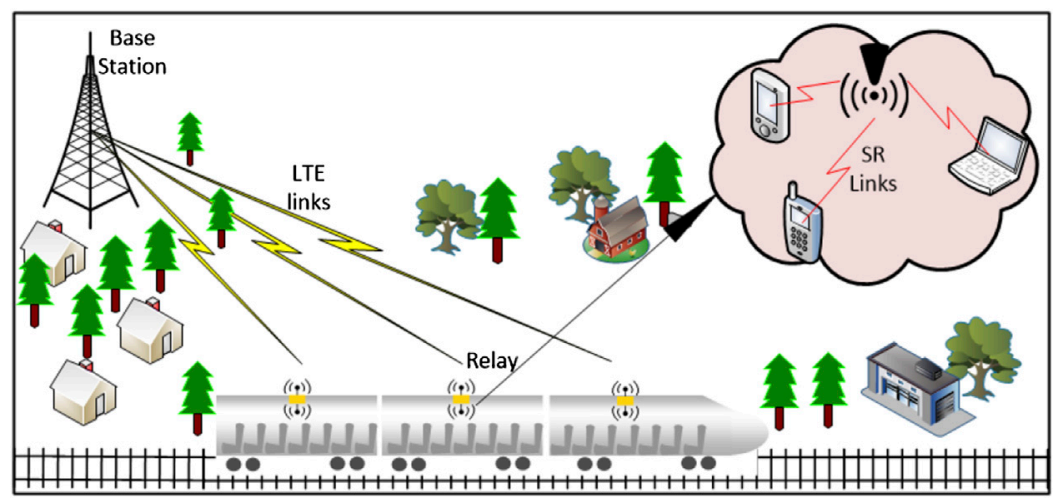

FIGURE 1 | Railroad connectivity with mobile relays.

with its outdoor antennas connected to the BS and its indoor antennas serving the UEs inside the wagon, where an indoor short range (SR) communication network is formed, as shown in Figure 1. The SR connectivity can be ensured through $\mathrm{WiFi}$, or through LTE/LTE-A femtocells for example.

This approach saves UEs from suffering large penetration losses in case of direct connection to the BSs, and spares them from being subjected to frequent handovers as the train moves at high speed. An additional benefit is also a reduced power consumption of the UEs, since it is less energy consuming to obtain the data via SR links with low mobility between UEs and MRs, than over LR links with frequent handovers at high train speed between the UEs and BSs. The scenario shown in Figure 1 can be enhanced when MRs are connected by fiber, and performance can be boosted by additional RRHs or small cells along the railroad track.

In this paper, we discuss 5G connectivity for railroad, analyzing both the passenger and train control communication networks. Challenges related to connectivity in railways are then listed, and appropriate solutions are outlined. We describe an approach for using MRs in conjunction with intelligent resource allocation, in order to meet the quality of service (QoS) requirements of passengers while minimizing the energy consumption of their UEs.

\section{G RAILROAD CONNECTIVITY}

A possible approach for designing railway communications consists of using a system for passenger data and another separate system for train control data. This latter system can be even split into two: one for mission critical data and the other for non-mission critical data. However, another approach consists of using one communication system (Gonzalez-Plaza et al., 2017). Indeed, with 5G, a single network can be used for all types of data with appropriate network slicing through software defined networking (SDN) and network function virtualization (NFV) (Hasegawa et al., 2018). This approach is preferable, since it allows the allocation of dedicated network resources for each scenario while using a unified and integrated physical architecture, which facilitates network planning, operation, and management.

5G defines three main use cases that all intersect with communications in modern railways:

- Enhanced mobile broadband (eMBB): This use case mainly handles passenger communications. It should cope with the tremendous increase in demand for high data rates due to the use of real-time video streaming, social media, large downloads, etc. It benefits from the presence of MRs to ensure high data rates inside the train while the outdoor antennas of the MRs ensure good connectivity with the BS and/or RRHs. In addition, with 5G, large numbers of antennas can be deployed to form massive multiple-input multipleoutput (MIMO) arrays. This is particularly useful for the BS-MR link since it can significantly boost performance. In addition, when MRs are deployed over each wagon as in Figure 1, they can be controlled by a single baseband unit (BBU) inside the train. Thus, their respective antennas can form a large MIMO array. When each MR is in turn equipped with its own MIMO array, then the joint operation of all the MRs can be identical to that of a large massive MIMO array controlled by the BBU, coupled with LOS with the BS, thus leading to huge communication data rates.

- Massive machine-type communications (mMTC): This use case is dedicated to accommodate a large number of sensing devices accessing the network frequently to send relatively short amounts of data. These could include sensors for monitoring the state of the railroad track, bridges, underground tunnels, etc. Their data can be aggregated locally (e.g., by a controller within the vicinity of a group of sensors) before being sent to the network. The collected data can be processed and analyzed on the servers of the company managing the railways, using data analytics and machine learning, in order to schedule periodic maintenance, predict the expected time before the need for repairs, and perform preventive maintenance, etc.

- Ultra-reliability and low-latency communications (URLLC): This use case corresponds to mission-critical services that do not tolerate delays. For example, it can consist of the 
TABLE 1 | Simulation parameters

\begin{tabular}{lc}
\hline Parameter & Value \\
\hline Train speed & $350 \mathrm{~km} / \mathrm{h}$ \\
Carrier frequency & $2.6 \mathrm{GHz}$ \\
Total system bandwidth & $20 \mathrm{MHz}$ \\
Total number of UEs & 250 \\
Total number of UEs per wagon & 25 \\
Transmit peak power at each MR & $20 \mathrm{dBm}$ \\
Transmit peak power at the BS & $46 \mathrm{dBm}$ \\
Shadowing standard deviation & $8 \mathrm{~dB}$ \\
Perpendicular train penetration loss & $18 \mathrm{~dB}$ \\
Parallel train penetration loss & $15 \mathrm{~dB}$ \\
Average noise power variance & $-174 \mathrm{dBm}$ \\
Average co-channel interference & $-110 \mathrm{dBm}$ \\
BS transmit antenna gain & $14 \mathrm{dBi}$ \\
MR transmit antenna gain & $0 \mathrm{dBi}$ \\
MR receive antenna gain & $0 \mathrm{dBi}$ \\
UE receive antenna gain & $0 \mathrm{dBi}$ \\
\hline
\end{tabular}

transmission of surveillance videos to take appropriate action in case of emergency. Another example consists of real-time control and management data, e.g., sending a command to stop a train due to an emergency, diverting a track into another route, etc. An appropriate 5G network slice should be dedicated to ensuring the required resources for URLLC. Furthermore, if needed in case of emergency, resources from the other slices should be allocated to the URLLC slice as it has the utmost priority.

Another aspect of $5 \mathrm{G}$ communications is the use of various frequency bands, with the use of millimeter wave (mmWave) frequencies being one of the most important 5G features. In (Yan et al., 2016), an integrated approach is presented where two types of RRHs are used: one type uses the licensed frequencies below $6 \mathrm{GHz}$, and the other type uses mmWave frequencies to meet the increasing capacity of user data. The simulation results of (Yan et al., 2016) showed an increase in capacity, especially in the vicinity of the RRHs.

However, propagation models for mmWave frequencies at high speed are not well-developed, and there are significant and important research efforts ongoing in this direction, e.g. (He et al., 2018), in order to characterize the propagation of these waves in different conditions (tunnels, rural and urban scenarios, straight and curved routes). Although they are suited for communication between RRHs and MRs, mmWave frequencies might be best suited for an mMTC slice dedicated for transmission of train control and management system (TCMS) information between sensors and RRHs (both fixed along the track, such that LOS can be easily maintained) or between sensors inside the train measuring train parameters and the internal train communication network (Gonzalez-Plaza et al., 2017). Sub-6Ghz bands can be used for longer range connectivity between MRs and macro BSs.

Next, we present seven major challenges faced by communications for railway systems. We focus on an energy efficient approach using MRs to meet the QoS requirements of train passengers, while minimizing the energy consumption at UEs and the transmit power at MRs and BSs. Thus, it mainly targets the $\mathrm{MBB}$ use case. However, the power savings at the BS and MRs can be diverted to support the operation of other slices corresponding to $\mathrm{mMTC}$ and/or URLLC as needed.

\section{DISCUSSION OF CHALLENGES AND POTENTIAL SOLUTIONS}

This section describes some important challenges faced by railway communications, and discusses potential solutions to address them. The results presented throughout this section are based on Matlab simulations using the parameters listed in Table 1 (Ghazzai et al., 2017).

\section{Doppler Effect}

Due to the high speed of the trains, Doppler effect cannot be neglected. In fact, it leads to high inter-carrier interference (ICI) in orthogonal frequency division multiple access (OFDMA) based systems. Doppler effect mitigation techniques are investigated in the literature, in addition to modified versions of OFDMA that lead to more robust behavior in face of the Doppler effect (Zheng et al., 2017). For example, the resource block (RB) filtered orthogonal frequency-division multiplexing (RB-F-OFDM) was shown in (Zheng et al., 2017) to outperform other waveforms. The results presented in this paper are based on allocating one $\mathrm{RB}$ per device and thus are easily applicable with RB-F-OFDM, although both RB-F-OFDM and the resource allocation algorithms used in this paper can be easily adapted to accommodate more RBs per device. Each RB in LTE consists of 12 consecutive OFDMA subcarriers, allocated for a transmission time interval (TTI) of $1 \mathrm{~ms}$. Another approach to deal with the Doppler effect is to benefit from the varying value allowed for subcarrier spacing (SPS) in 5G. In (Hasegawa et al., 2018), it was shown that SPS greater than $60 \mathrm{KHz}$ would be more suitable than the $15 \mathrm{KHz}$ used in LTE/LTE-A. Although the results would differ, most traditional radio resource management (RRM) algorithms designed for LTE/LTE-A can be applicable independent of the SPS, since they target the allocation of RBs irrespective of the SPS of the subcarriers in these RBs.

\section{RRM and Channel State Information (CSI) Feedback}

To perform efficient resource allocation, the BS needs to have relatively accurate channel state information (CSI). With the MR approach, the UEs and the MRs are at relatively fixed positions with low mobility with respect to each other, which solves the problem of having UEs continuously report to the BS CSI information that will arrive outdated due to the high speed of the train. However, there is still a need for CSI exchange between the MRs and the BS. A potential solution is the use of the "predictor antenna" method, e.g., see (Phan-Huy et al., 2015). Since the trajectory is well-known along the track, then, when several antennas are deployed on the train roof, a subsequent antenna will reach the position of the preceding antenna after a very short time period. The CSI sent by the first antenna will be irrelevant to that antenna when the BS makes and notifies the MR of its scheduling decision, but it will be highly correlated with the CSI of the subsequent antenna, since it would have reached the 


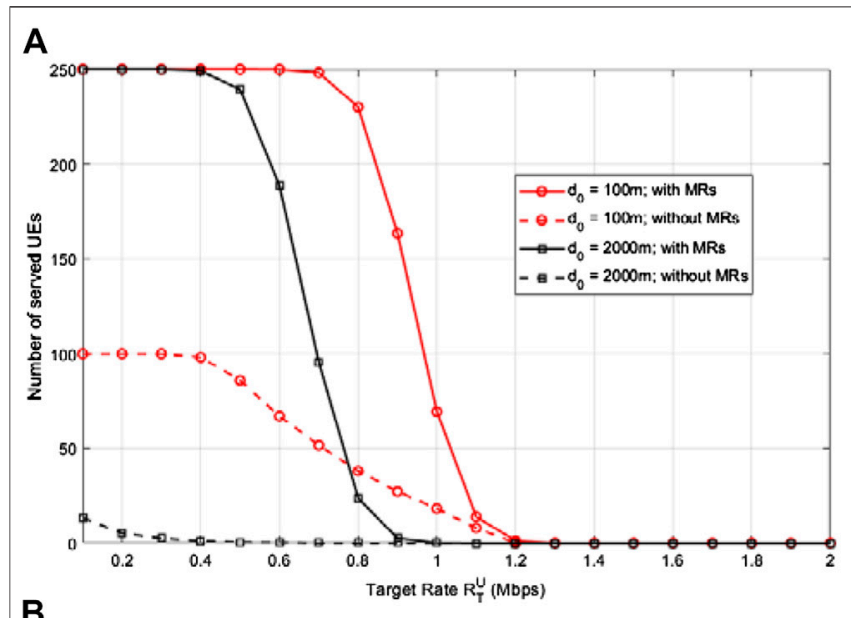

B

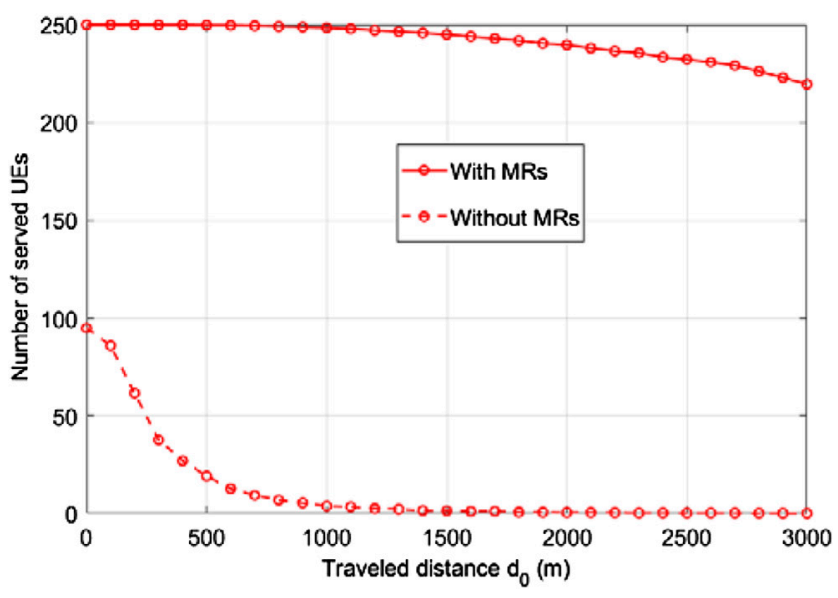

FIGURE 2 | Number of served UEs with and without MRs. (A) Total number of served UEs vs. $R_{T}^{U}$ for different distances. (B) Total number of served UEs vs. the distance between the BS and the train with a UE target rate of $\mathrm{R}_{\mathrm{T}}^{\mathrm{U}}=0.5 \mathrm{Mbps}$.

position of its preceding antenna. Thus, with this approach, a "dummy" antenna can be used to provide CSI for the first antenna element, and then the CSI of each antenna can be used for the antenna following it. This approach is feasible with 5G, especially with the adoption of massive MIMO techniques involving the use of large numbers of antennas. Each MR can possess a multitude of antenna elements placed on the roof of its wagon (e.g., patch antennas can be easily embedded within the structure of the wagon) that can be used with the predictor antenna method. Another method could be to use a "cross-slice" approach to avoid CSI feedback. In fact, the train position and speed are known accurately via feedback of GPS information in addition to sensor data from the rail track and onboard the train. This data communication is part of the mMTC slice, but if it is available at the BS, e.g., via edge caching techniques, then this information could be used for beamforming without the need for CSI. Indeed, the MRs with their antennas positioned outside the train will have a LOS link most of the time with the BSs deployed along the track (in tunnels RRHs can compensate for the lack of LOS). Thus, "geometrically" knowing the position of the train and its speed can allow the BS to perform beam steering with highly directive beams directed toward the MRs to boost the BS-MR signal (especially for the eMBB or URLLC slices), without traditional CSI feedback. Recent studies have already used the train position and speed information for beam switching (Cui et al., 2018).

Different intelligent RRM algorithms benefiting from the CSI information can be used. In this section, for illustration purposes, we use the Hungarian algorithm which provides an optimal solution for the assignment problem (assigning RBs to MRs on the LR BS-MR links and assigning RBs to UEs on the SR MR-UE links): On the BS-MR links, each MR is allocated a single $\mathrm{RB}$ that maximizes its performance. The same algorithm is implemented by each MR to perform RRM on the MR-UE links inside the train. Figure 2 shows the results of the outdoor BS-MR link for a train with ten wagons, along with 25 UEs per wagon. The total rate requested by each MR from the BS is the aggregate of the target rates requested by the UEs in its served wagon.

Figure 2A shows the number of served UEs vs. the target rate $\mathrm{R}_{\mathrm{T}}^{\mathrm{U}}$ required by each UE. It is noticed that all the UEs can be served up to a certain target rate, and the performance degrades afterward. This is due to the available power at the BS which, for a certain distance, can allow a maximum number of UEs to satisfy their QoS. As expected, a shorter distance to the BS will allow serving more UEs. In addition, Figure $\mathbf{2 A}$ shows that the performance degrades dramatically when no MRs are used.

Having RRHs installed along the railroad would have reduced the perceived distance with the BS and led to reduced power consumption. Furthermore, dynamic beamforming with massive MIMO antennas at the BS would have served the same purpose. These network design tradeoffs would depend on the access rights to the railroad, safety regulations allowing the installation of additional infrastructure, and cost constraints (Friedner et al., 2018).

In Figure 2B, the target rate per UE is set to $0.5 \mathrm{Mbps}$, such that the total rate requested by each MR from the BS is the aggregate of the target rates requested by the UEs in its served wagon. Thus, each MR would request a rate of $12.5 \mathrm{Mbps}$ from the BS. The results are plotted vs. the distance between the first wagon and the nearest BS. Most of the UEs are successfully served when MRs are used, with the number of served UEs decreasing slightly with the distance to the nearest BS. At $3 \mathrm{~km}$ separation, around 220 UEs out of 250 are served.

These results assume the MRs used are full duplex. In addition, these relays are decode-and-forward, which makes them suitable to scenarios where the outdoor (BS-MR communication) and indoor (MR-UE communication) technologies are different.

It should be noted that the results without MRs are dramatically worse. For example, in Figure 2B, for a distance of $100 \mathrm{~m}$ with the BS, around 90 UEs can be successfully served for a target rate of $\mathrm{R}_{\mathrm{T}}^{\mathrm{U}}=0.5 \mathrm{Mbps}$. This number reduces to less than five UEs when the distance reaches $2000 \mathrm{~m}$.

In addition, it should be noted that massive MIMO operation of MRs is not used in the presented results. Had the MRs been assumed to operate as a single $5 \mathrm{G}$ massive MIMO array under the control of a single BBU, along with the use of more bandwidth, then the data rate results would be orders of magnitude higher, since the MRs would be under a centralized control. The results 
corresponding to independent $\mathrm{MR}$ operation would thus constitute a lower bound on performance. Another interesting future research direction in this regard is the use of reconfigurable intelligent surfaces (Yang et al., 2020). They can be used to handle beamforming at the BS in the direction of the MRs. Moreover, if an RIS can be deployed on the outside area on top of the train wagons and connected to the MRs, then the performance would be significantly enhanced by using the RISs at both the train and BS.

\section{Handover}

When the UEs inside a train are connected directly to the BS, the handover of a large number of devices at high speed becomes a significant challenge. This problem is significantly alleviated by using MRs at the top of the train, although not necessarily at the frequency of $1 \mathrm{MR}$ per wagon. Since UEs are connected to MRs, the problem becomes that of handing over the MRs, i.e., a lower number of more powerful devices.

The handover problem in moving networks, especially in vehicular and public transport networks, has been studied extensively in the literature, e.g., (Morillo-Pozo et al., 2008; Araniti et al., 2009; Niyato and Wang, 2009; Trullols-Cruces et al., 2009; Borota et al., 2011; Sui et al., 2012a; Sui et al., 2012b; Sui et al., 2013). The handover with an MR on public transportation systems represents a complex scenario, since in these systems numerous macro, micro and picocells are installed at different urban locations, which might affect the handover. For example, a UE close to a bus window might have better reception from a picocell at a street pole nearby than from the indoor MR. However, the main challenge in trains is that the handover occurs at significantly higher speed, which increases the probability of dropped calls, which is significantly alleviated by using MRs in trains (Li et al., 2012).

Another scenario where Doppler and handover represent serious challenges is in unmanned aerial vehicle (UAV) networks. The mobility and flight altitude of UAV systems have a severe impact on their networking compared to traditional Mobile Ad Hoc Networks (MANETs) and Vehicular Ad Hoc Networks (VANETs). This motivated the investigation of networks of UAVs in the literature (Bekmezci et al., 2013; Gupta, et al., 2015; Zhou et al., 2015; Wang et al., 2017). In (Zhou et al., 2015), a twolayer erial-ground cooperative networking architecture was proposed, where multiple UAVs forming an aerial subnetwork assist a terrestrial vehicular subnetwork through UAV-to-UAV and UAV-to-ground communications, thus acting as intermediate relays. The multi-UAV system was first proposed in (Bekmezci et al., 2013) based on the concept of Flying Ad Hoc Network (FANET), where the network-centric methodology allowed the UAVs to optimize their positioning for enhanced connectivity and increased coverage due to cooperation with the other UAVs. This problem is less severe in trains since their location and route information can be more accurately acquired, and BSs can be deployed along the track.

Thus, using MRs in trains with indoor connectivity provides a solution to the UE handover problem at high speed. In addition, the more powerful MRs are less prone to outage and dropped connections since they can have better sensitivity than UEs,

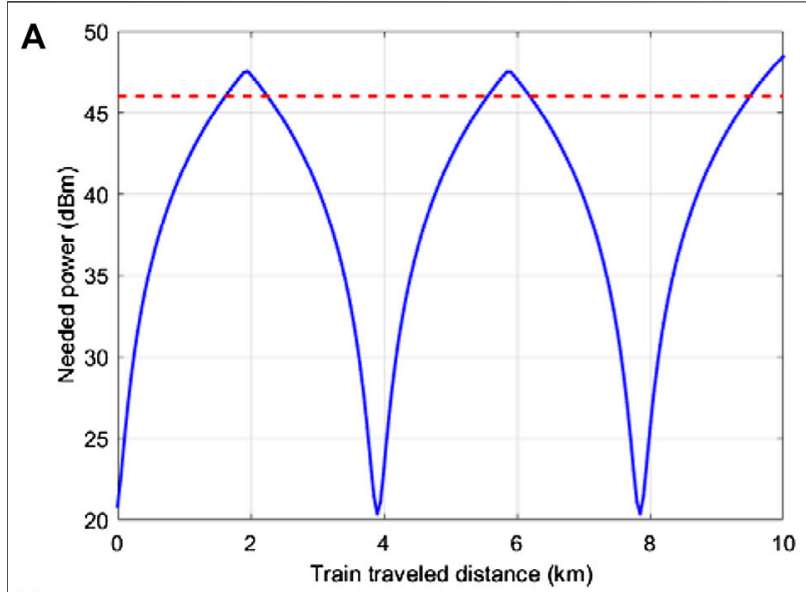

B

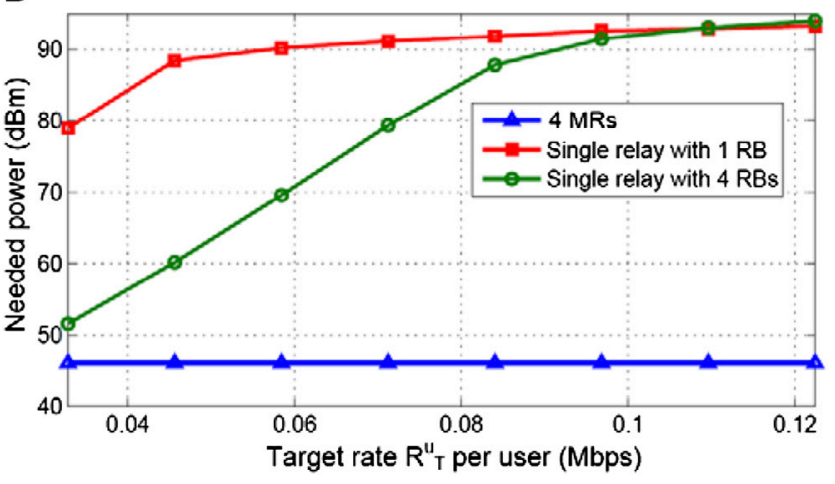

FIGURE 3 | BS power consumption example and the impact of MRs, assuming $46 \mathrm{dBm}$ is the total power available at the BS. (A) Total required power consumption in a high-speed railway with non-optimal planning. (B) BS power consumption comparison between the single relay and the moving relays scenarios.

higher transmit power, and the handovers can be pre-prepared in a train network since the positions of the BSs are known.

In fact, in (Xie et al., 2016), a concept similar to MRs is adopted where each train is equipped with two antennas: one at the front and the other at the rear. One antenna might perform a handover while the other is still connected to the initial BS in order to ensure a smooth transition to the users. Once the first handoff is successful, the second antenna can be handed over. A similar approach was adopted in (Zhao et al., 2018), while taking into account the details of handover between macrocell BSs (used mainly for train control data), or between micro BSs (used for user traffic) under the coverage of the same macro BS. This approach is extended in (Banerjee et al., 2018) to a situation where multiple antennas (more than two) are available on the train top. With the first-in-first-out (FIFO) approach of (Banerjee et al., 2018), whenever an antenna succeeds in performing a handover from one small cell to another, then the other antennas communicate with it internally over the train's fiber network. The switching occurs between antennas depending on which antenna is first successfully handed over to the target BS.

Handover performance can be further enhanced by power adjustment as suggested in (Lu et al., 2017). Under this approach, 


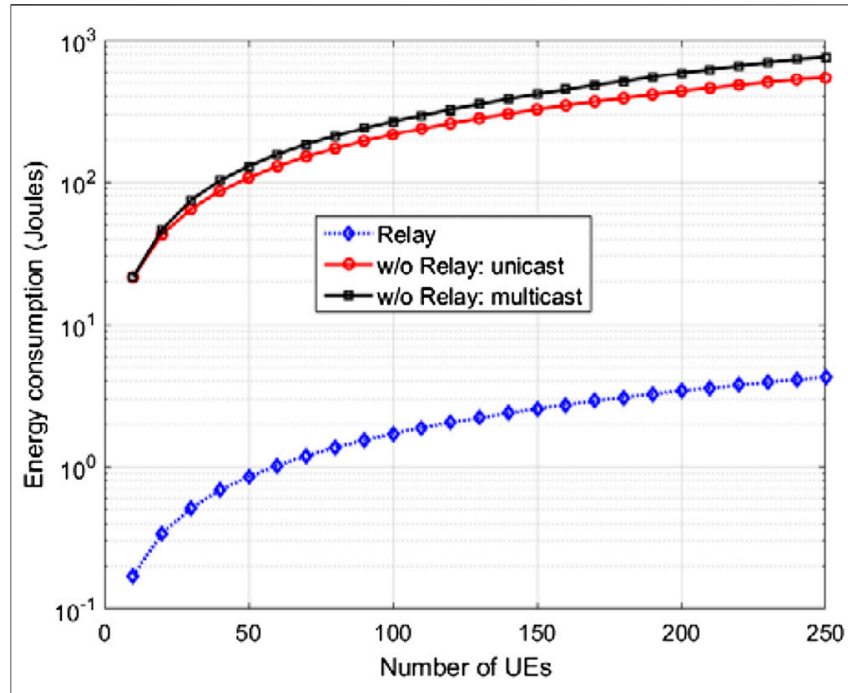

FIGURE 4 | Energy consumption of UEs in Joules vs. the total number of UEs when each wagon is equipped with a MR, such that the MRs are connected to LTE BSs outside the train and using WiFi inside the train.

when the train enters the overlap area between the serving cell and target cell, the serving cell increases its transmit power in order to delay the handover (thus avoiding premature handovers) until the train becomes closer to the target cell. At that moment, the target cell starts increasing its power whereas the serving cell decreases its power so that the handover can smoothly take place.

Thus, efficient planning of BS deployment along the track is important in order to optimize the handover operation, which is facilitated by the presence of MRs on the train wagons. Figure 3 shows an example corresponding to three BSs with inter-BS spacing of $3.8 \mathrm{~km}$. It is assumed that the total available transmit power at each BS is $46 \mathrm{dBm}$. Figure 3A shows that the needed power to achieve the QoS required by the UEs in the train can exceed this maximum power (represented by the horizontal dashed line), especially when the train is near the cell boundary, almost half way between consecutive BSs. When the planning process takes into account the presence of MRs in addition to the QoS requirements, significant enhancements can be obtained as shown in Figure 3B, where the presence of 4 MRs on the train leads to significant reduction in power consumption at the BS, with an inter-BS separation of $3.8 \mathrm{~km}$. Significant savings are obtained compared to the one MR scenario, even when the number of allocated RBs is increased to four to equal that of the four MRs case (since according to the simplified assumptions of the previous section, no more than one $\mathrm{RB}$ is allocated per MR).

\section{Energy Efficient Operation}

Energy efficient operation of the railway communications networks is often overlooked in the literature, where the focus is on topics of higher priority in these networks. However, careful planning of the network, in conjunction with intelligent resource allocation, can still lead to energy efficiency while maintaining safety and critical network operation. For example, small cells along the track dedicated purely to eMBB can be put into sleep mode when there are no trains passing. This process can be easier to implement in railroad networks, due to their nature and the predictability/beforehand knowledge of train trajectories, compared to, for example, BS on/off switching to save energy in "traditional" cellular networks, e.g., (Yaacoub, 2017). The efficient RRM and planning methods, e.g., (Ghazzai et al., 2017), and the results described in this paper (Figures 2, 3) can lead to energy savings that can be directed to other slices when needed (e.g., URLLC). In addition, even if all BSs are active, reduced power consumption due to intelligent resource allocation can lead to cost savings for the operators on the long run.

Figure 4 shows the total energy consumption of UEs in a train with ten wagons, along with 25 UEs per wagon, as per the parameters of Table 1 . The results shown correspond to receiving $1 \mathrm{Mbits}$ per each UE. The scenarios with and without MRs are compared. The multicasting scenario corresponds to a situation where the UEs are receiving the same content from the BS, e.g., the live broadcast of a football match. When the wagons are equipped with a MR, it is connected to an LTE BS with a total bandwidth of $5 \mathrm{MHz}$, corresponding to 25 resource blocks (RBs).

It is possible to use the same technology indoor and outdoor when MRs are deployed. In fact, LTE/LTE-A could be used inside the train wagons while the MRs can also be connected to the BS using LTE/LTE-A, as assumed in Table 1. This approach can be extended to $5 \mathrm{G}$ using OFDMA or any accessing schemes that will be adopted as enhancements to OFDMA. In such a scenario, the MR would act similarly to an indoor femtocell connecting the UEs inside each wagon to the network. Appropriate frequency subdivision of the various subcarriers should be performed between the indoor and outdoor portions of the network. This can be easily supported through carrier aggregation while benefiting from the fact that the indoor transmissions of each MR can be confined to its wagon to minimize interference.

Nevertheless, in Figure 4, it is assumed that each MR communicates with the LTE BS outside the train, and provides indoor WiFi connectivity to the UEs inside the train. Due to avoiding penetration loss and providing SR connectivity to UEs, the energy savings are large. For example, the total consumption for $200 \mathrm{MTs}$ is around $3.5 \mathrm{~J}$ compared to 430 and $600 \mathrm{~J}$ for unicasting and multicasting without MRs, respectively. This underlines the large energy savings for the UEs that can be reached when MRs are deployed. Although this scenario shares some similarities with indoor UEs in traditional cellular networks or energy-constrained Internet of things (IoT) devices, the problem in railroad networks is more challenging. Indeed, without MRs, the indoor UEs moving at high speed have to be connected to outdoor BSs, and suffer from frequent handovers and the impacts of Doppler effect. However, using MRs provides them with a short-range stable connection at higher data rates and no handovers, whereas the more powerful MRs, connected to the train power (and thus having no battery depletion problem), handle the communication with the outdoor BSs, the handovers, and the mitigation of Doppler issues. 


\section{Public Safety}

Public safety in railroad networks encompasses two main categories: 1) faults and issues along the rail track or problems in the train, e.g., fault monitoring along the rail or failures of train equipment, and 2) safety and protection from malicious users, e.g., burglars and terrorists.

The first type requires information exchange between terrestrial control centers and trains, such as information on the location of the train, its schedule, its state, level crossings, permitted speed, etc. Other information is related to the railroad status, e.g., autonomous track geometry measurement system (ATGMS) that can allow detecting existing defects along the rail line in real-time. These measurements can be transmitted using mMTC communications in 5G. However, when an emergency is detected, an alert should be sent with the utmost priority, which falls under the URLLC use case. In that case, the adopted RRM approach should cater for this kind of emergencies, and if needed, "steal" or puncture resources from eMBB users (e.g., see (Al-Ali et al., 2020)), which are typically the UEs of train passengers.

In addition to mission critical transmissions from railroad monitoring sensors in case of emergency, video surveillance transmission is an important factor for maintaining security in trains. Surveillance cameras deployed in train wagons have to send their monitoring videos to a control center. This imposes a significant load on the network. However, with the advances in video coding, e.g. through the use of High Efficiency Video Coding (HEVC), video can be coded in a scalable way with varying data rates (Yaacoub et al., 2015). For example, by varying the video coding parameters, the data rate can be made to vary between $64 \mathrm{kbps}$ and 2 Mbps. Thus, the high quality videos can be locally processed on a dedicated server in the train using artificial intelligence/machine learning techniques to detect any problems, e.g., by analyzing the behavior of passengers from the recorded videos and detecting normal/abnormal situations. In parallel, these videos can be transmitted to the control center at low data rates with "reasonable" quality in normal situations. Whenever an emergency is detected, the transmitted video quality can be increased along with an alert message to the control center. Appropriate resources should be reserved in the wireless network to maintain a "basic" operation of this network. However, whenever an emergency arises, resources can be diverted from the slice dedicated to passenger transmissions as needed to provide the utmost priority for the safety network.

\section{Security}

The data transmitted by the sensors that are part of the railroad management and control network should be secured against malicious attacks. Due to the nature of these sensors, it might be difficult to implement advanced cryptographic techniques. Therefore, physical layer security methods and/or lightweight encryption techniques are a suitable solution to address this problem while adapting to the constraints of the sensing devices (Liu et al., 2018). "Physical" attacks, e.g., sabotage, over these sensors can be mitigated either by surveillance cameras, and/or by allowing these sensors to transmit an alert when they suffer from sudden malfunction. This should be given the utmost priority under the URLLC use case, as discussed in the previous section, before the communications component of the sensor suffers permanent damage. Nevertheless, more advanced cryptographic techniques can be implemented to secure the video surveillance data discussed in the previous subsection, in order to protect the privacy of the passengers and the integrity of the data sent to the public safety control center. This surveillance data can be encrypted, and in case of attackers trying to capture this surveillance data and receive information about the train passengers, additional, physical layer security methods could be used to combat eavesdropping (thus maintaining privacy) or jamming (aiming to prevent the data from reaching the control center).

\section{Laws and Regulations}

Implementing a state-of-the-art communication network for railroad communications is not only dependent on pure technical aspects. In addition to determining whether a single network should be used for passenger communications and rail control/management or two separate networks, other details should be addressed. For example, it has to be decided if the network deployed will be under the control of a mobile network operator, of a dedicated service provider, of the train operating company, etc. If it is not under the control of the railroad authorities, appropriate rights and permissions should be granted to allow the communications service provider or network operator to access the properties of the rail network and install the needed infrastructure (Friedner et al., 2018). Furthermore, the equipment installed should take into account the rail safety standards and not hinder the operation of the trains. Thus, careful coordination and smart decision making should take place to avoid any bureaucratic hurdles that might affect the efficient deployment and operation of the railroad communication network.

Other issues related to law and regulations include the seamless transition of a train crossing networks of different countries or entities with a different law and regulations framework. Roaming agreements need to be set in place while trains cross seamlessly boundaries between different countries, as in the EU for example. Even when the countries do not share open borders, UEs at the Frontier of a country can be connected to the BSs of a network in a neighboring country. When UEs are connected to an MR and are seamlessly handed-over from one network to another (in a different country), high roaming charges might apply and this should taken into account by alerting the users connected to the train network. In addition to trains, the scenario of high roaming charges that could surprise the users also applies in the case of boats (BBC News, 2014; Schlichtkrull, 2017). In (Schlichtkrull, 2017), an example is described for satellite connectivity for boats between Denmark and Germany, where passengers were surprised by the high roaming fees while the boat was in International waters (for a short duration), as they assumed they were connected to either Danish or German operators with acceptable roaming prices.

\section{CONCLUSION}

In this paper, seven main challenges faced by modern $5 \mathrm{G}$ railway communication systems were reviewed and discussed. Suitable solutions to these challenges were outlined. A summary of the challenges and proposed solutions is presented in Table 2. In 
TABLE 2 | Summary of challenges and solutions.

\begin{tabular}{|c|c|}
\hline Challenge & Potential Solution(s) \\
\hline Doppler effect & $\begin{array}{l}\text { Using RB-F-OFDM and/or } \\
\text { Varying SPS }\end{array}$ \\
\hline RRM and channel state information (CSI) feedback & $\begin{array}{l}\text { Using intelligent RRM with: } \\
\text { Predictor antennas for CSI, and/or } \\
\text { Beamforming based on train location information } \\
\text { Using massive MIMO and RIS }\end{array}$ \\
\hline Handover & Group handover through the use of MRs: MRs are handed over between BSs while UEs remain connected to MRs \\
\hline Energy efficient operation & $\begin{array}{l}\text { Connect the UEs to the MRs from inside the train through indoor short range connectivity while the MRs handle long } \\
\text { range communication with the BS. }\end{array}$ \\
\hline Public safety & $\begin{array}{l}\text { Using URLLC for TCMS alerts and emergencies } \\
\text { Surveillance camera with intelligent RRM and video coding }\end{array}$ \\
\hline Security & $\begin{array}{l}\text { Physical layer and lightweight security techniques for securing sensor data } \\
\text { Encryption of transmitted surveillance videos }\end{array}$ \\
\hline Laws and regulations & $\begin{array}{l}\text { Roaming agreements between operators } \\
\text { Informing passengers on train of border crossings }\end{array}$ \\
\hline
\end{tabular}

particular, the use of mobile relays in conjunction with intelligent resource allocation was analyzed in terms of energy efficiency and enhanced connectivity. The discussed solutions for the other challenges constitute interesting topics for additional research investigations.

\section{AUTHOR CONTRIBUTIONS}

EY handled most of the write-up, in addition to Challenges in Sections Energy Efficient Operation, Public Safety, Security, Laws

\section{REFERENCES}

Al-Ali, M., Yaacoub, E., and Mohamed, A. (2020). "Dynamic resource allocation of eMBB-uRLLC traffic in 5G new radio." in IEEE international conference on advanced networks and telecommunications systems (ANTS), December 22, 2020, Online.

Araniti, F., Sanctis, M. D., Spinella, S., Monti, M., Cianca, E., Molinaro, A., et al. (2009May). "Cooperative terminals for incident area networks." in Proc. 1st international conference on wireless communication, vehicular technology, information theory and aerospace and electronic systems technology. 2009 Wireless VITAE'09, Aalborg, Denmark, May 17-20, 2009, 549-553.

Banerjee, S., Rakshit, S. M., Hempel, M., and Sharif, H. (2018). 5G-UCDA in high speed rail with multi antenna-to-logical cell circular FIFO mapping strategy. International conference on computing, networking and communications (ICNC), Maui, Hi, March. 5-8, 2018.

BBC News. (2014). "Faster internet on trains, boats and planes on horizon." Available at: https://www.bbc.com/news/technology-25825694 (Accessed November 26, 2020).

Bekmezci, I., Sahingoz, O. K., and Temel, S. (2013). Flying ad-hoc networks (FANETs): a survey. Ad Hoc Netw. 11 (3), 1254-1270. doi:10.1016/j.adhoc. 2012.12.004

Borota, D., Ivkovic, G., Vuyyuru, R., Altintas, O., Seskar, I., and Spasojevic, P. (2011). "On the delay to reliably detect channel availability in cooperative vehicular environments," in Proc. 73rd IEEE Vehicular Technology Conference VTC Spring 2011, Yokohama, Japan, May. 15-18, 2011

Cui, Y., Fang, X., Fang, Y., and Xiao, M. (2018). Optimal nonuniform steady mmWave beamforming for high-speed railway. IEEE Trans. Veh. Technol. 67 (5), 4350-4358. doi:10.1109/TVT.2018.2796621 and Regulations. AA worked mostly on the part described in RRM and Channel State Information (CSI) Feedback. HG worked mostly on the part described in Handover. M-SA supervised the whole work, and reviewed the write-up of the paper

\section{ACKNOWLEDGMENTS}

The authors would like to thank the Editor and the Reviewers for their comments that helped in enhancing the quality and clarity of this paper.

Friedner, S., Womersley, R., and Treacher, T. (2018). Connected train and customer communications: rail and digital industry roadmap. Rail Safety and Standards Board (UK), Technical Report,

Ghazzai, H., Bouchoucha, T., Alsharoa, A., Yaacoub, E., Alouini, M. S., and AlNaffouri, T. (2017). Transmit power minimization and base station planning for high-speed trains with multiple moving relays in OFDMA systems. IEEE Trans. Veh. Technol. 66 (1), 175-187 doi:10.1109/TVT.2016.2542344

Gonzalez-Plaza, A., Moreno, J., Val, I, Arriola, A., Rodriguez, P., Jimenez, F., et al. (2017). "5G communications in high speed and metropolitan railways." in 11th European Conference on Antennas and Propagation (EUCAP), Paris, France, March 19-24, 2017.

Gupta, L., Jain, R., and Vaszkun, G. (2015). Survey of important issues in UAV communication networks. IEEE Commun. Surv. Tutor. 18 (2), 1123-1152. doi:10.1109/COMST.2015.2495297

Hasegawa, F., Taira, A., Noh, G., Hui, B., Nishimoto, H., Okazaki, A., et al. (2018). High-speed train communications standardization in 3GPP 5G NR. IEEE Commun. Mag. 2 (1), 44-52. doi:10.1109/MCOMSTD.2018.1700064

He, D., Ai, B., Guan, K., Zhong, Z., Hui, B., Kim, J., et al. (2018). Channel measurement, simulation, and analysis for high-speed railway communications in 5G millimeter-wave band. IEEE Trans. Intell. Transport. Syst. 19 (10), 3144-3158. doi:10.1109/TITS.2017.2771559

Kim, J., Schmieder, M., Peter, M., Chung, H., Choi, S.-W., Kim, I., et al. (2019). A comprehensive study on mmWave-based mobile hotspot network system for high-speed train communications. IEEE Trans. Veh. Technol. 68 (3), 2087-2101. doi:10.1109/TVT.2018.2865700

Li, W., Zhang, C., Duan, X., Jia, S., Liu, Y., and Zhang, L. (2012). "Performance evaluation and analysis on group mobility of mobile relay for LTE advanced system." in Proc. of the 76th IEEE vehicular technology conference (VTC Fall'2012), Quebec, Canada, September 3-6, 2012. 
Liu, Z., Liu, J., Zeng, Y., and Ma, J. (2018). Covert wireless communications in IoT systems: hiding information in interference. IEEE Wirel. Commun. 25 (6), 46-52. doi:10.1109/MWC.2017.1800070

Lu, Y., Xiong, K., Fan, P., Zhong, Z., and Ai, B. (2017). The effect of power adjustment on handover in high-speed railway communication networks. IEEE Access. 5, 26237-26250. doi:10.1109/ACCESS.2017.2775044

Morillo-Pozo, J., Trullols, O., Barcelo, J., and Garcia-Vidal, J. (2008). "A cooperative ARQ for delay-tolerant vehicular networks." in Proc. 28th international conference on distributed computing systems workshops, ICDCS 2008, Beijing, China, June 17-20, 2008, 192-197.

Niyato, D., and Wang, P. (2009). Optimization of the mobile router and traffic sources in vehicular delay tolerant network. IEEE Trans. Veh. Technol. 58 (9), 5095-5104. doi:10.1109/TVT.2009.2025379

Phan-Huy, D.-T., Sternad, M., and Svensson, T. (2015). Making 5G adaptive antennas work for very fast moving vehicles. IEEE Intell. Transp. Syst. Mag. 7 (2), 71-84. doi:10.1109/MITS.2015.2408151

Schlichtkrull, J. (2017). Boats, Planes and Satellites: A Beginner's Guide to NonTerrestrial Roaming. Available at: https://www.roccoresearch.com/2017/09/27/ boats-planes-and-satellites-a-beginners-guide-to-non-terrestrial-roaming/ (Accessed November 26, 2020).

Sui, Y., Papadogiannis, A., and Svensson, T. (2012a). "The potential of moving relays - A performance analysis." in Proc. of the 75th IEEE vehicular technology conference (VTC Spring'2012), Yokohama, Japan, May 6-9, 2012

Sui, Y., Papadogiannis, A., Yang, W., and Svensson, T. (2012b). "Performance comparison of fixed and moving relays under co-channel interference." in IEEE Globecom Workshops (GC Workshops 2012), Anaheim, CA, December 3-7, 2012.

Sui, Y., Ren, Z., Sun, W., Svensson, T., and Fertl, P. (2013). "Performance study of fixed and moving relays for vehicular users with multi-cell handover under cochannel interference." International conference on connected vehicles and expo (ICCVE 2013), Las Vegas, NV, December 2-6, 2013.

Trullols-Cruces, O., Morillo-Pozo, J., Barcelo, J., and Garcia-Vidal, J. (2009). “A cooperative vehicular network framework." in Proc. IEEE international conference on communications (ICC 2009), June14-18, 2009, Dresden, Germany.

Wang, J., Jiang, C., Han, Z., Ren, Y., Maunder, R. G., and Hanzo, L. (2017). Taking drones to the next level: cooperative distributed unmanned-aerial-vehicular networks for small and mini drones. IEEE Veh. Technol. Mag 12 (3), 73-82. doi:10.1109/MVT.2016.2645481
Xie, S., Yu, X., and Luo, L. (2016). "A seamless dual-link handover scheme with optimized threshold for C/U plane network in high-speed rail.” in IEEE 83rd vehicular technology conference (VTC Spring 2016), Nanjing, China, May 15-18, 2016.

Yaacoub, E., Filali, F., and Abu-Dayya, A. (2015). QoE enhancement of SVC video streaming over vehicular networks using cooperative LTE/802.11p communications. IEEE J. STSP. 9 (1), 37-49. doi:10.1109/JSTSP.2014.2330343

Yaacoub, E. (2017). RESCUE: renewable energy small cells for utility enhancement in green LTE HetNets. IEEE Syst. J. 11 (4), 2356-2365. doi:10.1109/JSYST.2015. 2496230

Yan, L., Fang, X., Li, H., and Li, C. (2016). “An mmWave wireless communication and radar detection integrated network for railways." in IEEE 83rd vehicular technology conference (VTC Spring 2016), Nanjing, China, May 15-18, 2016

Yang, Z., Xu, W., Huang, C., Shi, J., and Shikh-Bahaei, M. (2020). Beamforming design for multiuser transmission through reconfigurable intelligent surface. IEEE Trans. Commun. 1. doi:10.1109/TCOMM.2020.3028309

Zhao, J., Liu, Y., Gong, Y., Wang, C., and Fan, L. (2018). A dual-link soft handover scheme for $\mathrm{C} / \mathrm{U}$ plane split network in high-speed railway. IEEE Access. 6, 12473-12482. doi:10.1109/ACCESS.2018.2794770

Zheng, Q., Wang, F., Chen, X., Liu, Y., Miao, D., and Zhao, Z. (2017). "Comparison of $5 \mathrm{G}$ waveform candidates in high speed scenario" in URSI General Assembly and Scientific Symposium (GASS), Montreal, QC, Canada, August 19-26, 2017.

Zhou, Y., Cheng, N., Lu, N., and Shen, X. S. (2015). Multi-UAV-Aided networks: aerial-ground cooperative vehicular networking architecture. IEEE Veh. Technol. Mag. 10 (4), 36-44. doi:10.1109/MVT.2015.2481560

Conflict of Interest: The authors declare that the research was conducted in the absence of any commercial or financial relationships that could be construed as a potential conflict of interest.

Copyright (c) 2021 Yaacoub, Alsharoa, Ghazzai and Alouini. This is an open-access article distributed under the terms of the Creative Commons Attribution License (CC $B Y)$. The use, distribution or reproduction in other forums is permitted, provided the original author(s) and the copyright owner(s) are credited and that the original publication in this journal is cited, in accordance with accepted academic practice. No use, distribution or reproduction is permitted which does not comply with these terms. 